\title{
Editorial
}

\section{A journey towards zero: malaria elimination in Sri Lanka}

\section{KDN Prasad Ranaweera}

Anti-Malaria Campaign, Ministry of Health, Sri Lanka

Correspondence:kdnpr1@gmail.com

DOI: https://doi.org/10.4038/jccpsl.v27i2.8449

Received on 2 June 2021

Accepted on 7 June 2021

Malaria is one of the major global public health problems at present (1). Malaria has been endemic in Sri Lanka for many centuries. However, Sri Lanka succeeded in becoming the second country in the World Health Organization (WHO) South-East Asia Region to receive WHO certification of malaria elimination(2).

Malaria control and elimination efforts in Sri Lanka are a rich tapestry of experience which spans over decades. It has been a journey full of experience from which many lessons can still be learnt. This success story has its roots firmly based on well-timed decisions, honest efforts and robust monitoring of actions. Albeit it is a cautionary tale, laying out the dire consequences of relaxing the efforts. This journey is about how Sri Lanka, despite a threedecade conflict and a low health budget, eliminated malaria and still continues to do so (3).

The first malaria control measures in Sri Lanka were initiated in 1910. However, more organized malaria control activities in the form of a 'campaign' were initiated in 1911 with the establishment of the 'Anti Malaria Campaign Centre' to cover the town of Kurunegala (4). Several major malaria epidemics have been recorded in the past, of which the most devastating one occurred in 1934-1935 affecting 1.5 million individuals and resulting in 80,000 reported deaths within a population just exceeding 6 million. The epidemic was contained through larviciding (oiling of rivers and streams, application of Paris green), quinine treatment and chemoprophylaxis. In November 1945, dichlorodiphenyltrichloroethane (DDT) spraying was initiated for the first time. From 1947 onwards, intensive indoor residual spraying (IRS) led to a drastic decrease in the cases. By late 1950s, the Government decided to engage in a malaria eradication programme in the context of the Global Malaria Eradication Programme launched by the WHO. In 1961, malaria was identified as a notifiable disease. A full-scale attack phase with blanket DDT spraying in endemic areas, accelerated malaria surveillance, intensified treatment with chloroquine and reporting procedures were conducted leading to a drastic reduction in the incidence. In 1963, only 6 out of the 17 reported cases were locally acquired (indigenous). This was the first-time the country had reached near elimination levels (4).

Due to several reasons including withdrawal of DDT spraying, insufficient surveillance in the scattered residual foci in jungle areas and lack of sustained funding, a resurgence of malaria occurred between 1967-1971 causing a massive epidemic with a peak of 538,000 cases being reported by 1969 . Almost all cases $(99.9 \%)$ were caused by P. vivax. This epidemic was contained by scaled-up vector control (reintroducing DDT) and surveillance interventions. In 1969 however, DDT resistance was reported. With failure of the elimination attempt, Sri Lanka resumed a malaria control policy in 1972 of case detection and 
treatment with chloroquine and primaquine. The $2^{1 / 2}$ decades that followed saw worsening of malaria throughout the endemic world. Global enthusiasm to control malaria waned after the "failed" Global Malaria Eradication effort of the 1960's and with this, international funding for malaria decreased significantly. Technical challenges of drug and insecticide resistance emerged and spread throughout the malaria endemic parts of the world. In 1984, chloroquine resistance in $\mathrm{P}$. falciparum was first reported in the country and by mid-1990s, the firstline treatment for falciparum malaria was changed to sulfadoxine/pyrimethamine. In 1994, Sri Lanka adopted the revised WHO Global Malaria Control Strategy, giving more importance to early diagnosis and prompt treatment, with mobile malaria clinics being scaled up between 1998 and 1999. Malathion was used for IRS from 1977 onwards, but resistance was detected in 1992 and pyrethroids (lambdacyhalothrin) were introduced. From 1996, vectorcontrol strategy was changed from universal coverage to targeted spraying in high-risk areas. Yet malaria epidemics continued to occur (4).

Sri Lanka embarked on the next major attack against malaria in 1999 when the WHO launched the Global Initiative to Roll Back Malaria in 1998. With this initiative, global support to malaria control increased greatly. Sri Lanka qualified for funding from the Global Fund to Fight AIDS, Tuberculosis and Malaria (Global Fund) established in 2002. New and improved tools became available and bold new strategies were recommended by the WHO. Distribution of long-lasting impregnated nets (LLINs) commenced in 2004 and more intense larval control measures were initiated. Treatment protocols were in line with the latest WHO recommendations based on parasite resistance data. The first-line treatment for P. falciparum infections was changed from previously single drugs to artemisinin-based combination therapy (ACT) in 2008. With increased funds being available, intervention coverage was scaled up (5).

Due to intensive Anti-Malaria Campaign (AMC) interventions, a dramatic drop in the number of cases and malaria incidence began in 2000. Even though Sri Lanka had achieved pre-elimination status by
2004/5 as defined by the WHO, the country was hesitant to embark on a plan for elimination due to the ongoing civil war in the country. This stable trend of decreasing malaria incidence continued in the subsequent years and between 2006 and 2010, the incidence was maintained at a very low level (0.010.03 per 1000 population). By 2007, there had been no deaths due to indigenous malaria in the country and after the conflict ended in May 2009, Sri Lanka embarked on the Malaria Pre-elimination Phase in September 2009 with financial assistance from the Global Fund. It was mandatory for all P. falciparum cases to be hospitalized for a minimum of three days since 2006, which in later years, became applicable for $P$. vivax cases as well. The policy of case reporting within 24 hours was established in 2009 \& a toll-free malaria hotline was introduced in 2010. Case reporting from the private sector was strongly facilitated since 2008. A stable shrinking of the malaria map was made possible, and the last major focus of P. vivax was in Hambantota (2009-2011) in a cluster of military camps located in and around the Yala National Park which was successfully controlled. In 2012, the number of indigenous cases dropped to 23. The last indigenous case was reported from the Victory Army Hospital, Anuradhapura in October 2012.

Effective vector control, epidemiological surveillance and early diagnosis and treatment contributed greatly to the success of malaria elimination in Sri Lanka, as did the contribution from all the regional malaria officers in endemic districts whose intensive efforts culminated in malaria elimination. Surveillance included active- and passive-case detection (ACD and PCD, respectively) enabling timely detection of cases; prompt and adequate treatment in accordance with national policies and guidelines; augmenting of ACD through mobile malaria clinics; strengthened laboratory support with quality assurance/quality control (QA/QC) procedures being conducted for confirmation of every clinical case of malaria; comprehensive epidemiological investigation of cases and foci to plan the response; strengthened and improved malaria information system facilitating quick decision-making; and regular monitoring of changes in the level of malaria receptivity and vulnerability. Integrated and cost-effective vector- 
control and entomological surveillance played an important role through integrated vector management with rational use of insecticides in rotation for IRS limited to areas with continued transmission, larviciding, LLINs, environmental modifications including the use of larvivorous fish. Intensive entomological surveillance provided important information on mosquito species, density, bionomics and breeding sites which were vital for planning effective vector control (5).

With the last case of indigenous malaria being reported in November 2012, Sri Lanka transitioned from the Elimination Phase to the Prevention of Reintroduction (PoR) Phase of malaria. Since then, all detected cases undergo confirmation and are classified according to WHO defined criteria as imported, indigenous, introduced, etc., and is reviewed by an independent panel of experts on malaria which constitutes the Case Review Committee (CRC) of the AMC which has been functioning since 2009. Sustaining zero cases for more than three consecutive years, Sri Lanka was certified a malaria-free country by the WHO in September 2016.

Since elimination, Sri Lanka reports around 50 imported malaria cases in the country every year. These and the presence of malaria vectors in many parts of the country constitute a risk of re-establishing malaria in the country. In the PoR phase, PCD is being continued, supported by ACD (reactive and proactive) and screening high-risk populations has become an important component of the surveillance system. Today, almost all the detected cases are treated, notified and investigated within 24 hours. Free chemoprophylaxis for Sri Lankans travelling to endemic countries ensured that the risk of importing malaria to the country was reduced. This is largely successful due to the strong collaboration with a number of local partners such as the tri-forces, Sri Lanka Police, private sector employers, relevant State ministries, departments and international partners. All regional malaria offices (RMO) are stratified based on the vulnerability \& receptivity which facilitate decision making on rational vector control. Vector surveillance and to a lesser extent vector control activities are being conducted, and include larviciding using locally available larvivorous fish, LLINs and conducting environmental management(5).

Lessons learnt in the past show that neglect of malaria interventions at this stage may once again result in the re-establishment and a rapid resurgence of malaria, requiring substantial efforts and financial support to combat. For Sri Lanka to remain malaria-free, current efforts should continue at the required momentum with sustained investments for strengthened case and entomological surveillance and response. It is the aspiration of all those at AMC and all healthcare workers in Sri Lanka that we continue to keep the country malaria-free.

Acknowledgements: Professor Kamini Mendis, Dr Hemantha Herath, Dr Ruwanika Seneviratne, Dr Priyanganie Silva

\section{References}

1. WHO. World Malaria Report 2020, Global Malaria Programme. Geneva: World Health Organization, 2020. ISBN 978-92-4-001579-1. Available from: https://www.who.int/publications/i/item/97892400 15791.

2. WHO. Vaccination requirements and recommendations for international travellers and malaria situation per country - 2019 edition. Geneva: World Health Organization, 2019. Available from: https://www.who.int/publications/ $\mathrm{m} /$ item/vaccination-requirements-andrecommendations-for-international-travelers-andmalaria-situation-per-country-2019-edition.

3. WHO. Malaria Free Sri LankaSouth-East Asia Region: World Health Organization, 2016. ISBN 978-92-9022-542-3. Available from: http:// apps.who.int/iris/bitstream/handle/10665/251824/ 9789290225423-eng.pdf;jsessionid $=4 \mathrm{~F} 5$ A72 BE5FF7DEDAB7D340EEEF8827E5? sequence $=1$

4. Fernando P \& Warusavithana S. 100 years of Malaria Control Efforts in Sri Lanka - 1911-2011. Colombo: M\&S Associates, 2011. ISBN 978-95553353-0-0.

5. WHO. Sri Lanka free of malaria: case study. Regional Office for South-East Asia: World Health Organization, 2017. Available from: https:// apps.who.int/iris/handle/10665/272396.

Journal of the College of Community Physicians of Sri Lanka 